\title{
La espiritualidad de la unidad en san Ignacio de Antioquía: una propuesta de vida cristiana"
}

\author{
Juan Camilo Rada Pardo**
}

Recepción: 30 de mayo de 2018 • Aprobación: 28 de junio de 2018

\section{Resumen}

La vida cristiana está íntimamente penetrada por la presencia de Dios que abarca toda la existencia. San Ignacio de Antioquía ofrece un itinerario espiritual centrado en la unidad por la que se interpreta cómo actúa esta infusión de Dios. La pertinencia de la vivencia mística cristiana es algo preeminente en la noción de Iglesia en salida como interiorización de la unidad del cuerpo místico de Cristo o como la necesidad de la experiencia comunitaria de la evangelización. La mística de la unidad fundamentada en la unidad de Dios y vivida en la comunidad eclesial es una propuesta de vida coherente con el misterio de Cristo, una opción para vivir auténticamente la vocación cristiana.

Palabras clave: unidad, san Ignacio de Antioquía, vida cristiana, Iglesia en salida.

* Artículo de investigación producto del semillero de investigación Gnosis: antigüedad cristiana y estudios patrísticos, que fue presentado en el I Coloquio Interinstitucional de Estudiantes de Patrología el 25 de mayo de 2018 en la Pontificia Universidad Javeriana de Bogotá. Citar como: Rada Pardo, J. C. (2019). La espiritualidad de la unidad en san Ignacio de Antioquía: una propuesta de vida cristiana. Albertus Magnus, X(2), 107-117. DoI: https://doi.org/10.153322/5005413.5215.

** Universidad Santo Tomás, Bogotá, Colombia. Orcid: https://orcid.org/0000-0002-77390452. Correo electrónico: juanradap@usantotomas.edu.co 


\title{
The spirituality of the unity in saint Ignatius of Antioch: A proposal for a christian life
}

\begin{abstract}
The Christian life is intimately penetrated by the presence of God that embraces all of existence. Saint Ignatius of Antioch offers a spiritual itinerary centred on the unity by which this infusion of God is interpreted. The relevance of the Christian mystical experience is something preeminent in the idea of a Church, which goes forth, either as an internalization of the unity of the mystical body of Christ, or because of the need for the community experience of evangelization. The mystique of unity based on the unity of God and lived in the ecclesial community is a proposal of life coherent with the mystery of Christ, an option to authentically live the Christian vocation.
\end{abstract}

Keywords: unity, Ignatius of Antioch, Christian life, Church that goes forth.

\section{A espiritualidade da unidade em santo Inácio de Antioquía: uma proposta para uma vida cristã}

\section{Resumo}

A vida cristã é intimamente penetrada pela presença de Deus que abrange toda a existência. Santo Inácio de Antioquía oferece um itinerário espiritual centrado na unidade pela qual esta infusão de Deus é interpretada. A relevância da experiência mística cristã é algo preeminente na idéia da Igreja em saída, seja como uma internalização da unidade do corpo místico de Cristo, seja por causa da necessidade da experiência comunitária de evangelização. A mística da unidade baseada na unidade de Deus e vivida na comunidade eclesial é uma proposta de vida coerente com o mistério de Cristo, opção de viver autenticamente a vocação cristã.

Palavras-chave: unidade, Inácio de Antioquía, vida cristã, Igreja em saída. 


\section{Introducción}

El ser cristiano implica una adhesión profunda a la persona de Jesús, un itinerario de seguimiento que empapa toda la vida del creyente y lo motiva a salir de sí para encontrarse con Dios y con el prójimo. No obstante, ante los insistentes llamados a la unidad que Francisco ha hecho durante su magisterio, siempre con la visión de una Iglesia en salida, la teología de la unidad de san Ignacio de Antioquía se muestra una propuesta de seguimiento de Cristo que sirve de molde para la vivencia de la fe. Una invitación que abarca el aspecto personal y comunitario de la vida creyente, siempre impulsado por la iniciativa de Dios, quien fue el que nos llamó a estar con Él primero. La unidad compete a todos los cristianos en vista de que permite la acogida de Cristo para todos, de hecho, es la unidad lo que permite ser fermento de amor para el mundo (Francisco, 2013a, n. 246).

Para san Ignacio de Antioquía, la unidad es un tema transversal en su obra. Por supuesto, el ambiente particular de las Iglesias de Asia Menor y de Roma permea la intencionalidad del autor en la escritura de las cartas, que, por su género literario específico, no pretende hacer una exposición dogmática elaborada, pero, aun así, el eje central de su obra es este, hasta el punto de que el mismo emisor se presenta como "hombre dispuesto a la unidad" (ad Phil. VIII,1). A pesar de los pocos testimonios para abordar la vida y teología de san Ignacio, su vida también permite entrever el misterio de la unidad en su persona. El obispo de Antioquía parece que no habla solo con su autoridad episcopal cuando se dirige a las diferentes Iglesias (ad Rom. IV, 3), sino probablemente poniéndose a sí mismo como ejemplo a las comunidades (ad Eph. III,1).

De la vida de Ignacio, obispo de Antioquía, se sabe relativamente poco. Fue condenado a morir a manos de las fieras en tiempos del emperador Trajano (98117), pero no en su ciudad sino que fue trasladado prisionero a Roma. En su camino a la Ciudad Eterna, Ignacio aprovecha para escribir siete cartas, de las que cinco están dirigidas a las Iglesias de Asia (Éfeso, Magnesia, Tralia, Filadelfia y Esmirna), una a Policarpo, obispo de Esmirna, y una última a la comunidad cristiana de Roma. El acercamiento a la mencionada teología de la unidad de san Ignacio se ha hecho a partir del estudio directo de estas cartas, así como de algunos de los documentos académicos más recientes sobre el obispo de Antioquía.

La metodología para abordar la mística de la unidad de Ignacio parte de una aproximación a los fundamentos teológicos: la unidad de la Trinidad inmanente, de la carne y el espíritu de Cristo, y de Cristo con su Padre. En un segundo momento, entender cómo la unidad de Dios sirve de arquetipo para la unidad de los creyentes con su Dios, unidad que se mantiene en la fe y en el amor. Por último, 
ver la unidad de la Iglesia con su Dios en virtud de la Pascua de Cristo, la unidad de las Iglesias en la catolicidad de la gran Iglesia y de la unidad intraeclesial, en torno a su obispo, por la solidaridad de sus miembros y como expresión del amor de Dios (Aguirre, 2010). Las conclusiones versarán sobre el constante llamado de Francisco a la unidad y cómo es que la oferta de san Ignacio es tan válida hoy como lo fue en el primer siglo de la Iglesia.

\section{Iniciativa de Dios para mostrarnos su vida de unidad}

Las raíces teológicas de la mística de la unidad se entierran en la misma naturaleza de Dios. Aunque no es necesario profundizar en la naturaleza monoteísta del Dios cristiano, sí es menester acercarnos a la naturaleza del Hijo. Cristo, Hijo de Dios, aun en su naturaleza humana, nunca perdió la unidad con su padre celestial, hecho testimoniado por los Evangelios que demuestran la íntima relación del Hijo con su Padre, especialmente en la obediencia a su palabra (ad Eph. XVIII,2), pero también en los signos que mostraron la procedencia divina de su poder. Esta misma obediencia es la que lo llevó a su entrega en la cruz, sufrir su pasión por redimir a la humanidad (ad Phil. VIII).

También es fundamental la unidad del ser de Jesucristo, quien, siendo verdaderamente Dios, no abandona su naturaleza divina, tras la kenosis. La unidad indisoluble entre la humanidad y divinidad de Cristo en su vida terrena es la que fundamenta la posterior unidad de la divinidad y humanidad de la Iglesia. "Nuestro señor es verdaderamente de la estirpe de David, según la carne, hijo de Dios por la voluntad y el poder de Dios" (ad Smyr. I,1-2).

La divinidad del Hijo se mantiene unida a la del Padre de modo que es verdaderamente efectiva la relación filial que posteriormente se dará, de forma análoga, entre la Iglesia y su Dios. Esta unidad espiritual es evidenciada, incluso, en la eucaristía, entendida, para Ignacio de Antioquía, como la efectividad del sacrificio pascual de Cristo dentro de la comunidad eclesial (Zañartu, 1982a).

\section{Respuesta del creyente que se une a Dios}

De la unidad de Cristo con su Padre los cristianos también pueden hacerse partícipes. En virtud de que el sacrificio de redención acoge en filiación adoptiva a todos los fieles, la relación de Cristo con su Padre es análoga a la relación de los 
fieles con Dios. Sin embargo, esta unidad únicamente se hace posible en la Iglesia que representa la unidad mayor de todos los fieles con su Padre. Los fieles se unen a Dios por medio de la unión de estos con Jesucristo.

En este sentido, por la fe y el amor, el cristiano mantiene la unidad a la divinidad y a la humanidad de Cristo: "unirse al Cristo de carne y espíritu, a su humanidad y divinidad, a su muerte y resurrección: todo el misterio de la encarnación redentora" (Trevijano, 2009, p. 36). Fue el amor lo que motivó la kenosis del Verbo: que el Invisible se hiciera visible por la humanidad y que, finalmente, soportó todo por nosotros (cfr. ad Pol. III,2), de modo que corresponde al cristiano encaminarse en el amor para llegar a Dios (ad Eph. IX,1), como lo hizo Cristo, por medio de la solidaridad con los hermanos de la comunidad (ad Smyr. VI,2), pero trascendiendo, incluso, eso, ya que el amor es, en últimas, lo que dinamiza la vida creyente. Asimismo, la fe se convierte en lo que sostiene la vida del cristiano (ad Tral. VIII,1), la sustancia de la vida cristiana (Bower, 1974, p. 10), de modo que el fiel asuma en su ser la cruz de Cristo por el Espíritu Santo.

La unidad del cristiano con Cristo se traduce en la adhesión profunda a su persona. "Toda la vida del cristiano debe tender a imitar y reproducir esta unidad de carne y espíritu realizada en Cristo, esa misteriosa unidad de Cristo con el Padre" (Trevijano, 2009, p. 37). En este sentido, la unión con Cristo es también sumisión decidida a la palabra de Dios Padre. La intención final es ser imitadores de Cristo, como él lo fue del Padre (Quasten, 2004, p. 35), asumir en la propia vida la misma vida de Jesús de Nazaret.

Consecuentemente con el seguimiento, es inevitable recorrer el camino de la pasión y de la cruz del Señor. La imitación de Cristo hasta la pasión no es en virtud de una pasión ilusoria, fantástica o incorpórea, sino de una verdadera pasión acontecida en un verdadero hombre con carne verdadera (Barreiro, 2002). Si no hubiesen acontecido los hechos realmente, la vida del cristiano también fuera una ilusión, una vaga e infundada opción de vida y, por ende, irreal. El sufrimiento en la carne de Cristo debe ser entendido, por tanto, no en el sentido paulino de debilidad, sino en el sentido joánico de la encarnación (Barreiro, 2002, p. 252). Más adelante se mencionará la pertinencia de esta categorización en vista de la doble influencia ignaciana.

El martirio es la más perfecta imitación de la pasión de Cristo, ya que la muerte es el camino por medio del cual se puede "alcanzar a Dios" (ad Rom. IV,1). Quien quiera asemejarse al Señor, también debe querer el martirio como meta (Bower, 1974). El cristiano solo llega a la perfección de su discipulado al entregar 
su vida por el Nombre ${ }^{1}$ (Quasten, 2004). El martirio crea vínculos estrechos con la pasión de Jesús: por un lado, de solidaridad con esta que desborda los sentimientos de la persona hasta el punto de asumir en su propia vida la experiencia del sufrimiento de la cruz; también, el vínculo de la imitación, hecho que se fundamenta en la verdadera humanidad de Jesucristo: los cristianos asumen en su carne la realidad de Cristo sufriente; finalmente, el vínculo del seguimiento, puesto que la decisión de seguir a Jesucristo no se limita a la adhesión a su mensaje sino a la interiorización de su vida, entonces, no es posible que el martirio pase desapercibido (Ayán, 2010).

Cabe recordar que las cartas de san Ignacio fueron escritas de camino a su martirio en la capital imperial tras haber sido arrestado en Antioquía ${ }^{2}$. Si Ignacio escribe exhortando a las comunidades a seguir a Cristo hasta el derramamiento de sangre, lo hace mostrándose a sí mismo como cumplidor de esta característica del discípulo, hecho que le imprime grandeza a su enseñanza. El obispo de Antioquía es consciente del destino que le aguarda, y lo espera ansiosamente. Inclusive, Ignacio asume que la Iglesia de Roma tiene amplio poder para salvarlo del martirio que él había decidido. No es impensable que la Iglesia pudiera interceder para una apelación de ciudadano romano ${ }^{3}$ (Davies, 1976, p. 177). Pero su convicción le permite seguir su camino con la frente en alto. Se refiere al martirio diciendo: "Para mí es mejor morir para Jesucristo que reinar sobre los confines de la tierra. Busco a Aquel que murió por nosotros. Quiero a Aquel que resucitó por nosotros. Mi parto es inmanente" (ad Rom. VI,1). Y prosigue pidiéndoles a los romanos que no se opongan a su destino: "Perdonadme, hermanos. No impidáis que viva; no queráis que muera. No entreguéis al mundo al que quiere ser de Dios, ni lo engañéis con la materia" (ad Rom. VI,2).

Es evidente la influencia paulina y joánica de Ignacio. La misma cristología ignaciana está permeada de la vivencia en Cristo del cuarto evangelio,

1 Término usado en la literatura cristiana, principalmente en los textos apostólicos, para referirse al mismo Cristo (Ayán, 2010).

2 Las razones de su arresto y la posterior condena aún no están esclarecidas. Teniendo en cuenta que los testimonios sobre Ignacio de Antioquía versan en sus cartas y en menciones de autores muy posteriores (a pesar de la probable gran influencia que su obra tuvo en gran parte de la cristiandad temprana), se hace muy difícil establecer una biografía responsable, cuyos datos históricos se conozcan con exactitud. Aunque no es el tema principal en este momento, es importante considerar el hecho de la prisión y el martirio que le espera.

3 Es importante el dato de las posibles influencias de la Iglesia de Roma en el poder imperial. Su primacía evidenciará cierto influjo de la comunidad de Roma con respecto a su sociedad y sobre las demás Iglesias. 
probablemente por el contexto gnóstico ${ }^{4}$, y de la corriente paulina, especialmente por la inmanencia de Dios en el alma humana (Quasten, 2004). Dios habita en el alma del creyente. Es muy interesante la adopción de términos como portador de Cristo o portador de Dios para referirse a esta inhabitación; de hecho, usa el seudónimo de Theoforo en todas las salutaciones epistolares. La unión, la vivencia y la imitación de Cristo hunde sus cimientos en la concepción firme de que la divinidad de Cristo habita en cada creyente como en un templo (Trevijano, 2009. p. 39). La inhabitación divina comporta un compromiso moral por parte del creyente: "Hagamos, pues, todo [con la conciencia] de que Él habita en nosotros, para que seamos templos suyos, y Él sea en nosotros nuestro Dios, tal como, en efecto, es y tal como aparecerá ante nosotros", y concluye afirmando que esta es la razón del seguimiento de la persona de Jesús: "Por ello justamente lo amamos" (ad Eph. XVI,3). Con todo, aunque la convicción de la presencia de Cristo es un tema cristocéntrico, no es meramente cristológico sino trinitario. Es para unirse también al Padre que sigue a Cristo (ad Magn. I,2) hasta entregarse a la radicalidad del martirio (ad Rom. VII,2). Pero esta unión, en virtud de Cristo, solo es posible si el Espíritu Santo es el que conduce al creyente a Dios (ad Eph. IX,1). En últimas, la vivencia de la unidad llevará a la vivencia comunitaria, paralelamente a la vivencia comunitaria de la Trinidad inmanente.

\section{La unidad de la Iglesia}

La unión eclesial es donde desemboca la mística de la unidad como manifestación mejor de la unidad de Dios y de la unidad de los creyentes con su Dios. Es la primera vez que en un texto aparece el nombre de "católica" referido a la Iglesia conformada por todas las iglesias locales. La garantía de unidad es el mismo Jesucristo, dado que su presencia en la Iglesia es la que garantiza su permanencia y acción en el mundo (ad Smyr. VIII,2). Cristo actúa en la Iglesia universal de forma análoga como lo hace el obispo en la comunidad local de modo que es eje

4 Algunos como Zeitschrift der Friedrich Schilier o Hans-Werner Bartsch comparten la idea de que toda la doctrina ignaciana, especialmente la de la unidad, tiene en sí una fuerte influencia gnóstica. Con mayor claridad, afirma este último la educación del obispo de Antioquía: primero de las primitivas comunidades cristianas, luego una influencia gnóstica indirecta recibida a través de los textos joánicos y, por último, un influjo gnóstico directo. El recurso joánico, finalmente, le sería útil para combatir las corrientes docetas y la teología judía, cuyas doctrinas atentaban contra la unidad de la naturaleza divina y humana de Jesucristo (Barnard, 1963, pp. 194, 198). 
de unión de la Iglesia e intermediario ante el Padre (Zañartu, 1982b, p. 7). La base teológica se encuentra en la misma unidad del Hijo con el Padre, para quien es la mente y el sentir de este, hecho que garantiza la unidad con la Trinidad.

Pero si Cristo es el centro de la unidad eclesial, existe una figura visible de la unidad de todas las Iglesias: la comunidad de Roma. Desde el saludo con el que Ignacio se dirige a los romanos en su carta, se evidencia un profundo respeto y una mención mucho más especial para con la comunidad: "ha alcanzado la misericordia en la magnificencia del Padre Altísimo y de Jesucristo, amada e iluminada en la voluntad del que ha querido todo lo que existe conforme al amor de Jesucristo". Además, prosigue con la mención explícita de la superioridad de la comunidad romana y por eso es "digna de Dios, digna de honor, digna de bienaventuranza, digna de alabanza, digna de éxito, digna de pureza", dado que, efectivamente, "está a la cabeza de la caridad", depositaria de la ley de Cristo y adornada con el nombre del Padre" (ad Rom. Int.). La Iglesia de Roma también tiene la autoridad para enseñar (ad Rom. III,1), porque su precedencia es en el amor y por ser el amor lo que dinamiza la vida de los creyentes y la esencia misma del cristianismo (Zañartu, 1982b).

Por otro lado, la Iglesia misma es continuadora de la obra de Dios en la historia de la salvación. Con su fundación, no ha acontecido una ruptura con el primer testamento, sino que lo plenifica con una singular superioridad (ad Phil. IX,2). Lo propio del Evangelio es el misterio de la pasión del Señor y la fe posterior que se desprende de la obra redentora de Jesús, entonces las tendencias judaizantes que permean las comunidades no son congruentes con la unidad que debe conservar la Iglesia por la fe en Cristo (ad Magn. X,3). A la Iglesia converge todo creyente en vista de que es culminante de la historia de la salvación, de hecho, los que se salvaron en la antigua Alianza fue por estar unidos a Cristo (Zañartu, 1982b).

En últimas, la unidad de la Iglesia es similar a la unidad de la naturaleza del Jesucristo, análogamente hablando, el cuerpo de la Iglesia son los fieles y su espíritu es Cristo mismo: la Iglesia se une a Dios por medio de Cristo, quien integra

5 La primacía de la Iglesia de Roma ha hecho que diversos autores inviertan tinta en su interpretación. Entre otros dilemas, una de las principales preguntas versa sobre si la comunidad romana goza de preeminencia universal o solo en un área geográfica determinada, posiblemente la misma ciudad. En este trabajo, se acoge la interpretación de la primacía universal por ser la que es más evidente al acercarnos a la obra ignaciana en general. Ante todo, se debe rechazar la interpretación de una doble intencionalidad de Ignacio de Antioquía al referirse con tan laudables términos a la comunidad de Roma. No es acertado pensar que el obispo de Antioquía recurra a la demagogia en la Carta a los Romanos para evitar la intervención de la Iglesia en un posible intento para rehuir su martirio (Zañartu, 1982b). 
el misterio de la unidad de Dios que funda la vida comunitaria (Zañartu, 1982b). Pero la unión no puede ser únicamente espiritual (unión con Dios por Cristo), sino que se hace patente en la unidad con el obispo (ad Magn XIII,2). Todo apunta a una dinámica ascendente: "Ignacio muestra un escalonamiento jerárquico de unión, que es continuo: los cristianos están íntimamente unidos al obispo, como la Iglesia a Jesucristo, y como Jesucristo al Padre; así todo es sinfónico en la unidad" (Zañartu, 1982b, p. 9). La carnalidad de la Iglesia exige la sumisión al obispo quien es imagen del Padre (ad Tral. III,1) y preside en su lugar (ad Magn. VI,1), porque el Padre es primer lugar, es el obispo de todos (ad Magn. III,1) y el obispo es "reflejo y manifestación" de Dios Padre, obispo invisible (Zañartu, 1982a, p. 4).

\section{Conclusión: espiritualidad de la unidad para una Iglesia en salida}

Es el Espíritu Santo el que permite la unidad en la Iglesia. Con la acción del Espíritu de Dios, es posible que en la diversidad de la Iglesia exista una unidad digna de admirar. Es más, la unidad propiciada por el Espíritu es la que nos lleva a la unidad de la Trinidad. Siempre es bueno recordar que la unidad no es sinónimo de uniformidad, el Espíritu permite que no se olvide ni el individuo cristiano ni la particularidad de cada Iglesia local. Una posible unidad elaborada por manos humanas muy seguramente llevará a la uniformidad que contraría a la multiplicidad de carismas de la Iglesia (Francisco, 2013a, n. 117). De hecho, es Cristo quien ha unificado todo en sí, por eso, es posible hacer el camino a la unidad (n. 228). Entonces, una espiritualidad de la unidad es querida, permitida, asistida y manifestada por Dios. La labor de los cristianos es recurrir al principio de la solidaridad para reconocer que la unidad es superior al conflicto (n. 228), que la llamada divina supera cualquier obstáculo de caos para transformarlo en orden, y que, si Dios quiere la unidad, los fieles hemos de procurarla.

De hecho, la desunión es antitestimonio de vida en Dios. Evidentemente algunos cristianos han dejado de ser fermento de unidad. Si se quiere ser cristiano, es necesario estar unidos al Señor. Si se quiere mostrar a Cristo, es necesario estar unido a Él. En efecto, la unidad facilita la acogida de la persona de Cristo, tanto para el fiel que ya camina en la imitación de Cristo como para el que lo conoce por vez primera (Francisco, 2013a, n. 246).

La intención es que todos los cristianos sean uno, concentrándose en las convicciones que los unen y recordando el principio de jerarquía de verdades (Francisco, 2013a, n. 246). Entonces, la propuesta de san Ignacio también puede 
ser aplicada en virtud del ecumenismo. Todas las Iglesias son necesarias, hecho fundamentado en las virtudes de la figura del obispo como eje de unidad de cada Iglesia local y como signo eficaz de capitalidad. La unión que permite el sacrificio eucarístico basta para garantizar la autoridad de todas estas, así como el reconocimiento de la comunión con otras Iglesias en la plenitud de la vida de fe y sacramental (Scampini, 2005).

La unidad de la fe permite una mejor evangelización. La confesión eclesial de la fe motiva la misión, puesto que la fe en la Iglesia es la que permite la auténtica exposición de Jesucristo en la historia dada la naturaleza espiritual y carnal de la Iglesia (Francisco, 2013b, n. 22). La unidad de fe, caridad y esperanza proyecta hacia un futuro cierto y seguro, lejos de parecerse a las predicciones ilusorias del mundo (n. 57), que bien podrían interpretarse como fermento de división de manera similar a como las heterodoxias lo fueron en su momento para Ignacio (ad Smyr. V,1).

Finalmente, una Iglesia que vive la unidad no puede sino ser fermento en todas las realidades terrenas, de modo que la unidad impregne todo el mundo. La unidad se establece como camino para la superación de todos los conflictos sociales; conflictos que generan división en la humanidad y que nacen de la mentalidad consumista devoradora (Francisco, 2013b, n. 198). El mundo entero clama por una luz de esperanza, una señal que le ayude a recobrar la fe en la humanidad misma, en la humanidad entera, en la humanidad unida. El desinterés de los poderosos del mundo frente a los conflictos sociales procede de una mentalidad de permanencia y autosuficiencia que parece ser contraria a cualquier vestigio de unidad en las sociedades. Que la Iglesia recuerde que la unidad es superior al conflicto es iniciar el camino de interacción entre todos los hombres que lleva a la consecución del bien común.

Lo que motiva al fiel cristiano a estar en constante salida es haber recibido el Evangelio del Señor, saberse encontrado por Dios primero (Francisco, 2013a, n. 3). La teología de la unidad parte de un fundamento en el que Dios es el que toma la iniciativa y permite que haya unidad. Así, el cristiano se siente unido a su Dios por la inhabitación del Señor en su ser, hecho fundamental para saberse cristiano y llenarse de la alegría, hasta el punto de asumir en su carne la misión de Jesucristo y seguirlo hasta el martirio. Por tanto, si Dios se ha llegado al creyente, este no puede sino responderle a través de la comunidad cristiana y del mundo, uniéndose profundamente al misterio de la Iglesia y a la misión concreta de ser signo de unidad para todas las naciones. 


\section{Referencias}

Aguirre, R. (ed.) (2010). Así empezó el cristianismo. Navarra, España: Verbo Divino. Recuperado de http://www.luisjovel.com/wp-content/uploads/2016/03/AguirreRafael-Asi-Empezo-El-Cristianismo.pdf

Ayán, J. J. (ed.) (2010). Padres apostólicos (2.ํe ed.). Madrid, España: Ciudad Nueva.

Barnard, L. W. (1963). The background of st. Ignatius of Antioch. Vigiliae Christianae, 17(4), 193-206. DOI: 10.2307/1582286

Barreiro, A. (2002). Os misteérios da vida de cristo nas cartas de santo Inácio de Antioquía e sua importáncia atual. Perspectiva Teológica, 34(93), 247-264. Recuperado de http://www.faje.edu.br/periodicos/index.php/perspectiva/article/view/634

Bower, R. A. (1974). The Meaning of $\dot{\pi} \pi \tau v \gamma \chi \alpha \dot{\alpha} \nu \omega$ in the Epistles of St. Ignatius of Antioch. Vigiliae Christianae, 28(1), 1-14. DOI: 10.2307/1583598

Davies, S. L. (1976). The predicament of Ignatius of Antioch. Vigiliae Christianae, 30(3), 175-180. DOI: $10.2307 / 1583332$

Francisco, (2013a). Evangelii gaudium. Ciudad del Vaticano: Editriche Vaticana.

Francisco, (2013b). Lumen fidei. Ciudad del Vaticano: Editriche Vaticana.

Francisco, (2015). Laudato si'. Ciudad del Vaticano: Editriche Vaticana.

Quasten, J. (2004). Patrología. Madrid, España: Biblioteca de Autores Cristianos.

Scampini, J. (2005). "La Iglesia” como tema teológico en el diálogo ecuménico. Vida Pastoral, 256. Recuperado de http://www.sanpablo.com.ar/vidapastoral/nota. php?id=191

Trevijano Etcheverría, R. (2009). Patrología (2. e ed.). Madrid, España: Biblioteca de Autores Cristianos.

Zañartu, S. (1982a). Algunas enseñanzas de Ignacio de Antioquía. Mensaje, 31(311), 401-404. Recuperado de http://repositorio.uahurtado.cl/static/pages/docs/1982/ n311_401.pdf

Zañartu, S. (1982b). Aproximaciones a la eclesiología de San Ignacio de Antioquía. Stromata, 38, 243-281. Recuperado de http://www.revistaapuntes.uc.cl/facteo/MaterialSergioZanartu/zanartu_web_2008/Internet_2007/IgnacioEclesiologia.pdf 